\title{
Absolute Pitch and Planum Temporale
}

\author{
J ulian Paul Keenan,* Ven Thangaraj,* Andrea R. Halpern, $†$ and Gottfried Schlaug* \\ *Department of Neurology, Beth Israel Deaconess Medical Center, 330 Brookline Avenue, Boston, Massachusetts 02215; and \\ †Department of Psychology, Bucknell University, Lewisburg, Pennsylvania 17837
}

Received February 20, 2001: published online October 24, 2001

An increased leftward asymmetry of the planum temporale (PT) in absolute-pitch (AP) musicians has been previously reported, with speculation that early exposure to music influences the degree of PT asymmetry. To test this hypothesis and to determine whether a larger left PT or a smaller right PT actually accounts for the increased overall PT asymmetry in AP musicians, anatomical magnetic resonance images were taken from a right-handed group of 27 AP musicians, 27 nonmusicians, and 22 non-AP musicians. A significantly greater leftward PT asymmetry and a significantly smaller right absolute PT size for the AP musicians compared to the two control groups was found, while the left PT was only marginally larger in the AP group. The absolute size of the right PT and not the left PT was a better predictor of music group membership, possibly indicating "pruning" of the right PT rather than expansion of the left underlying the increased PT asymmetry in AP musicians. Although early exposure to music may be a prerequisite for acquiring AP, the increased PT asymmetry in AP musicians may be determined in utero, implicating possible genetic influences on PT asymmetry. This may explain why the increased PT asymmetry of AP musicians was not seen in the group of early beginning non-AP musicians. 2001 Academic Press

Key words: music; absolute pitch; morphometry; planum temporale; laterality.

Absolute pitch (AP) is the ability to identify the pitch of any tone in the absence of a musical context or reference tone (Takeuchi and Hulse, 1993). The mechanism and neural basis underlying this ability remain unclear. There is evidence that AP is highly correlated with early exposure to music (Krumhansl, 1991; Miyazaki, 1988, 1989) and it has been suggested that AP manifests itself in a critical period of brain development (Sergeant, 1969; Schlaug, 1995a)

One region that is of interest in regard to AP is the planum temporale (PT), a region historically associated with language and auditory processing. The PT is found to be asymmetric in normal right-handed samples, with a greater leftward bias (i.e., left > right). The
PT has been frequently used as a marker of hemispheric dominance comparing both normal and clinical populations. Although there are several studies that have found differences in PT asymmetries depending on handedness (Foundas et al., 1994; Shapleske et al., 1999; Steinmetz et al., 1991; Tzourio et al., 1998), there is still an ongoing dispute in the literature over whether there is a gender effect in PT asymmetry (Foundas et al., 1999; Ide et al., 1996; J äncke and Steinmetz, 1993; Kulynych et al., 1994). Similarly, it is unresolved if PT differences exist between normal individuals and schizophrenics (Barta et al., 1997; Kleinschmidt et al., 1994; Shapleske et al., 1999) or between normal individuals and dyslexics (Leonard et al., 1993; Shapleske et al., 1999). Common to each of these studies are the behavioral implications of hemispheric asymmetries of brain regions implicated in language and, more general, in auditory processing.

In regard to AP, it has been suggested that there are differences in PT asymmetry between AP musicians and matched non-AP controls. Schlaug and colleagues (1995b) found a greater leftward PT asymmetry between AP musicians and non-AP musicians. Zatorre and colleagues (1998) found a greater leftward PT asymmetry when AP musicians were compared to a large control sample, although no significant differences in the asymmetry score were found between a small sample of AP and a small sample of non-AP musicians. These studies demonstrating a possible increased leftward asymmetry in AP musicians were intriguing, considering the usual association of musical processing with right-hemisphere activity changes in nonmusicians or individuals unselected for musical background (Zatorre et al., 1992, 1994). However, several studies questioned whether this association was true in musicians (Bever et al., 1974; Mazziotta et al., 1982; Besson et al., 1994).

These findings have raised a series of important questions. First, the covariation between a particular musical ability (i.e., AP) and the PT has been an unsuspected finding that needs to be replicated in a larger sample to address its consistency and further explore the nature of asymmetric PT differences. One impor- 
tant, yet not fully examined, possibility is whether the increased PT asymmetry in AP musicians is due to an increase in the left PT, a decrease in the right $\mathrm{PT}$, or a redistribution and reallocation of resources between the hemispheres. Galaburda and colleagues speculated that the right PT was critical in determining observed PT asymmetries (Galaburda et al., 1987; Galaburda, 1994). In terms of AP, Zatorre and colleagues (1998) found a significant difference in the left PT volume between a small group of AP musicians compared to non-AP musicians unselected for musical skill, but no significant difference was found for the right PT in those group comparisons. However, our previous study (Schlaug et al., 1995b) in a much smaller sample than reported in this study demonstrated a trend for a smaller right PT and a larger left PT, relative to both non-AP musicians and nonmusicians. This raises the possibility that hemispheric specialization might involve a redistribution of resources across the hemispheres and not just an expansion of one particular area.

Second, and equally important, is an understanding of the determinants of an increased leftward PT asymmetry in AP musicians. It is unknown whether the increased leftward asymmetry is due to early exposure to music and early musical training or is determined prenatally. It has been suggested that early musical exposure during critical developmental periods may lead to morphometric differences in motor-related brain structures (Schlaug et al., 1995a; Amunts et al., 1997). It is unknown whether early musical exposure or the AP ability per se led to morphometric differences. In order to determine whether early exposure to music might have an effect on PT asymmetry or PT sizes, we have tested two large control groups, one group of matched (handedness, age, and gender) nonmusicians and one group of early beginning musicians without absolute pitch but with a similar early commencement of musical training.

\section{METHOD}

\section{Subjects}

We examined a sample of 27 right-handed (12 M/15 $\mathrm{F}$; age $\mathrm{M}=26.74$ years, $\mathrm{SD}=6.73$ ) classically trained musicians (mainly keyboard players; some also played string instruments in addition) with self-reported absolute pitch ability (AP mus.). The first control group consisted of 27 right-handed nonmusicians (12 M/15 F ; age $M=24.89$ years, $S D=5.75)$. Most of the nonmusician controls (Nonmus.) were students at local colleges or universities or professionals in the medical field. Nonmusicians were defined as having had no formal training in music or never having played a musical instrument. All were newly recruited from the Boston area.
The second control group consisted of 22 righthanded (13 M/9 F; age $M=26.32$ years, $S D=9.94)$ non-absolute-pitch musicians (Non-AP mus.). The nonabsolute-pitch musicians were taken from a databank of over 70 musicians collected over the past 5 years. The selection criteria were non-absolute-pitch musician, right-handed, and commencement of musical training before the age of 7 . The matching for gender in this group was not optimal, but we have yet to find a gender effect in our group with regard to PT asymmetry (J äncke and Steinmetz, 1993), and a recent metaanalysis (Shapleske, 1999) did not find a significant difference in the absolute size nor in the differences between left and right PT size between men and women.

The absolute pitch and non-absolute-pitch musician groups were matched with regard to commencement of musical training (AP mus. $M=5.06, S D=1.14$, range 3-7 years of age; Non-AP mus. $M=5.09, S D=0.97$, range 3-7 years of age; $t(47)=-0.09, P>0.05)$. They were also matched in terms of the number of years of musical instrument experience (AP mus. $M=21.68$, $\mathrm{SD}=6.55$; N on-AP mus. $\mathrm{M}=21.23, \mathrm{SD}=9.92 ; \mathrm{t}(47)=$ $0.19, \mathrm{P}>0.05)$.

Right-handedness was confirmed in all subjects with standardized tests (Annett, 1992; J äncke et al., 1997). All of the subjects gave written informed consent to this study according to institutional guidelines.

\section{Absolute Pitch Tests}

Absolute pitch ability was assessed using two established tests (Ward and Burns, 1982; Zatorre and Beckett, 1989). In both tests, computer-generated sine wave tones with a duration of $500 \mathrm{~ms}$, and an attack and decay rate of $30 \mathrm{~ms}$, were presented binaurally. All tones corresponded to fundamental frequencies. Subjects were made familiar with the computer-generated sine wave tones by listening to a sample sequence consisting of 10 tones. The first AP test consisted of 13 tones taken from a chromatic scale ( $\mathrm{F} \# 3$ to $\mathrm{F} \mathrm{\# 4);} \mathrm{each}$ tone was repeated four times in random order with a 4-s interstimulus interval. Subjects were required to write down the tone name in the 4-s interstimulus interval. After completing the first run through the total of 52 tones, subjects had to listen to the tones a second time and were allowed to make corrections if needed. No reference tone was provided at any point during the tests and subjects were not informed about their performance.

The second AP test consisted of a total of 48 sine wave tones taken from 4 1/2 octaves (F 2 to G5). First, a set of 12 tones was generated, separated by a musical fourth ( $F 2, B b 2, E b 3$, etc.), so that there was no duplication of chroma across octaves, but large degrees of differences in tone height. The resulting 12 tones were presented four times in random order for a total of 48 
tones. Interstimulus interval was similar to the first test. Again, all subjects listened to the whole sequence of tones two times. In agreement with the literature (Miyazaki, 1988; Ward et al., 1996; Zatorre and Beckett, 1989), we regarded a response within $1 / 2$ tone difference of the presented tone as a correct response. In order to be regarded as an absolute pitch possessor, subjects had to have more than $90 \%$ correct of their responses on both tests. All of the musicians who claimed to have absolute pitch were confirmed by these tests (i.e., all AP musicians in this study scored $90 \%$ or higher correct responses on both of these tests). The mean correct responses were 50.5 (SD 2.1) for the set of 52 tones and 46.3 (SD 2.1) for the set of 48 tones. None of the non-AP musicians and none of the nonmusicians were able to perform these two tests, since they were not able to identify any of the tones without a reference tone which we did not provide. All non-AP musicians were allowed to listen to the tones, but they typically gave up after the first few tones, since they were not able to identify any of these tones correctly.

\section{MR Data Acquisition and Morphometric Analyses}

Magnetic resonance imaging was performed employing a T1-weighted magnetization prepared, rapid acquisition gradient-echo (MPRAGE) pulse sequence on a 1.5-T Siemens Vision MR system with echo-planar imaging capability (Siemens Medical Systems, Erlangen, Germany). A three-plane scout was acquired prior to the MPRAGE to ensure that the interhemispheric plane was aligned with the sagittal plane of imaging. In case this was not achieved, the subject was repositioned. The image dataset had a voxel resolution of $1.0 \times 1.0 \times 1.0 \mathrm{~mm}$ and provided us with excellent anatomical detail.

For offline morphometry, the datasets obtained from all groups were mixed and coded. The morphometric analysis was performed on a Hewlett-Packard workstation by one observer, blinded for musician or control and hemisphere. The intraobserver correlation coefficient (Pearson) on a subset $(n=15)$ of the subjects for JPT was 0.95 ( $\mathrm{P}<0.001)$. The interobserver correlation between the observer in this study and other observers in previous large sample studies on the PT was above 0.85 (J äncke et al., 1994; Schlaug et al., 1995b; Steinmetz et al., 1995).

The planum temporale was defined according to previously published criteria (Steinmetz et al., 1991; Schlaug et al., 1995b). In short, the observer had to identify the sagittal slice that contained the retroinsular origin of the anterior transverse gyrus of Heschl. This point defined the medial border of the PT and it was the point where the anterior and posterior borders of the PT coincided. Progressing laterally, the PT was determined on each subsequent slice by tracing a cursor manually within the gray matter of the posterior superior temporal plane from the anterior to the posterior border of the PT. The anterior border of the PT was defined as the bottom of Heschl's sulcus, the posterior border of the PT was defined as the transition point where the posterior descending ramus of the Sylvian fissure flips into the ascending ramus. This border is also classically defined by the "knife" criterion. The lateral border of the PT was defined as the lateral rim of the superior temporal plane (Steinmetz et al., 1989, 1990, 1991). In subjects in whom the anterior border of the PT did not continue up to the lateral border, a straight subsidiary line was used to complete the anterior border laterally. This subsidiary line started from the lateral end point of Heschl's sulcus and ran perpendicularly to the midsagittal plane.

In this study, we represented the planum temporale contours on sagittal slices as splines to approximate the curvature found in the gyral pattern of the brain (Bartels et al., 1989). To better determine the true surface area, a triangular mesh was generated between contours on consecutive slices based on the methods of Meyers and colleagues (1992). The cost function used to calculate the minimal spanning tree was modified to be a combination of area plus perimeter of each triangle. Surface area was then calculated as a sum of the area of all the triangles defining the surface of interest. The area of each triangle was the cross product of any two vectors defining the edges of the triangle.

Left-right asymmetry of the PT surface area was expressed as the asymmetry coefficient $\partial \mathrm{PT}$ ( $\mathrm{R}$ $L /(R+L) \times 0.5)$ with $R$ and $L$ being the surface areas of the right and left PT. Negative values indicate leftward asymmetry, and positive values indicate rightward asymmetry.

\section{Data Analysis}

A series of planned comparisons was employed using the General Linear Model. For JPT comparisons, a univariate analysis was performed, while left and right PT comparisons were made using multivariate tests. Post hoc comparisons were done for each contrast with Bonferroni corrections. To determine the effectiveness in group categorization, a binary logistic regression was employed for the two musician groups for both the left and the right PT. Data were also compared between the current sample and that previously reported by Schlaug et al. (1995b). All data were processed employing SPSS 9.0 for Windows.

\section{RESULTS}

Employing a univariate analysis on $\partial \mathrm{PT}$ average, it was found that there was a significant overall difference between the three groups in this study $(F(2,73)=$ 7.996, $P<0.001)$. The strength of this effect as indexed 


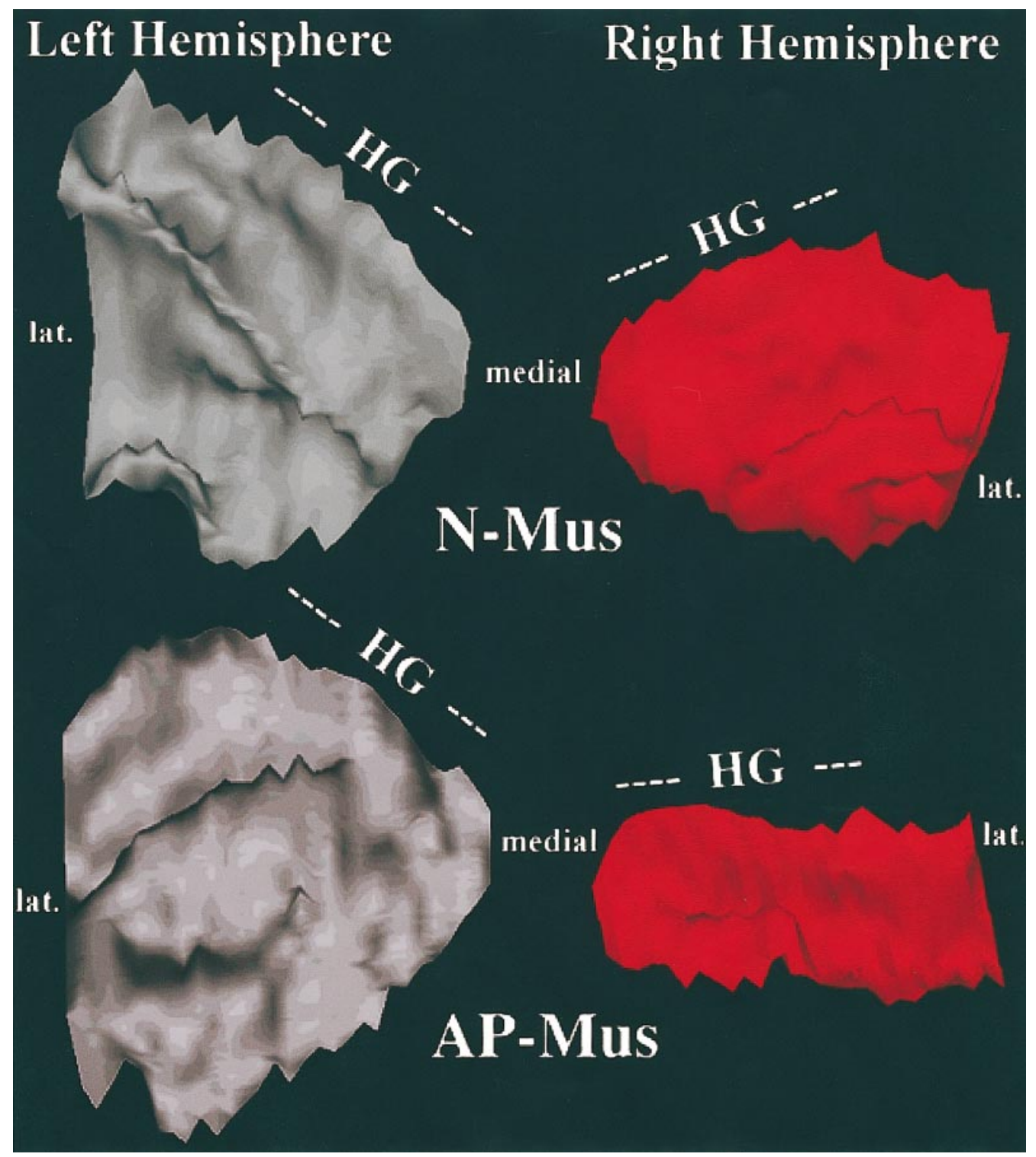

FIG. 1. Surface reconstructions of the right and left PT of one nonmusician and one musician with absolute pitch. The approximate location of the transverse gyrns of Meschl (MG) is indicated for each PT surface reconstruction. Note the large difference in right hemisphere PT size for these two subjects.

by $\eta^{2}$ was 0.180 . Corrected Bonferroni adjustments $(\mathrm{P} / 5)$ revealed that the AP mus. group differed significantly from both the Non-AP mus. group $(P<0.001)$ and the Nonmus. group ( $<<0.01$; Fig. 1). The Non-AP mus. group and the Nonmus. group did not differ significantly from each other ( $P>0.05$; Table 1$)$.

A breakdown of the $\partial \mathrm{PT}$ analysis was further performed across the three groups by examining the right and left PT. Employing a multivariate GLM, examining both right and left PT ( $\times$ Group), it was found that there was a significant difference for right PT $\left(F(2,73)=7.77, P<0.001 ; \eta^{2}=0.176\right)$, but not left PT $(F(2,73)=0.24, P>0.05)$. Bonferroni adjusted $(P / 5)$ comparisons revealed that, within the right $\mathrm{PT}$, the AP mus. group had a significantly smaller right PT compared to both the Non-AP mus. group ( $P<0.002$; Fig. $1)$ and the Nonmus. group $(P<0.006)$. The Non-AP mus. group and the Nonmus. group did not differ significantly from each other ( $P>0.05$; Table 1 ).

To determine the effect of both the left and the right $\mathrm{PT}$ on group, a binary logistic regression (AP and Non-AP musicians) was employed. First, the individual contributions were considered. The left PT model $\left(\operatorname{Mod} \chi^{2}(1)=\right.$ 0.40 ; Wald $=0.39, \mathrm{P}=0.53$; \%class $=51.02 \%$ ) did not significantly predict group membership while the right PT did $\left(\operatorname{Modx}^{2}(1)=13.23\right.$; Wald $=9.78, \mathrm{P}<0.002$; \%class $=73.47 \%$ ). However, the right PT model did significantly improve with the addition of the left PT added 
TABLE 1

Overall Surface Area and PT Asymmetry Scores: Means and Standard Deviations of the Three Groups

\begin{tabular}{|c|c|c|c|}
\hline & $\begin{array}{c}\text { Right PT }\left(\mathrm{mm}^{2}\right) \\
{[\mathrm{M}(\mathrm{SD}) \text { and range] }}\end{array}$ & $\begin{array}{l}\text { Left PT }\left(\mathrm{mm}^{2}\right) \\
\text { [M (SD) and range] }\end{array}$ & $\begin{array}{c}\partial \mathrm{PT} \\
{[\mathrm{M}(\mathrm{SD}) \text { and range] }}\end{array}$ \\
\hline AP mus. $(N=27)$ & $\begin{array}{l}828.17(232.61) \\
467.13 / 1408.40\end{array}$ & $\begin{array}{r}1401.55(444.71) \\
696.93 / 2731.73\end{array}$ & $\begin{array}{l}-0.498(0.27) \\
-1.04 / 0.06\end{array}$ \\
\hline Non-AP mus. $(\mathrm{N}=22)$ & $\begin{array}{r}1079.02(212.18) \\
742.85 / 1423.62\end{array}$ & $\begin{array}{r}1333.82(285.84) \\
936.40 / 2120.93\end{array}$ & $\begin{array}{l}-0.209(0.16) \\
-0.55 / 0.04\end{array}$ \\
\hline Non mus. ( $\mathrm{N}=27$ ) & $\begin{array}{r}1043.00(283.07) \\
592.22 / 1569.05\end{array}$ & $\begin{array}{c}1365.01(342.73) \\
897.29 / 1932.96\end{array}$ & $\begin{array}{l}-0.275(0.33) \\
-0.93 / 10.26\end{array}$ \\
\hline Overall & $\begin{array}{l}977.10(267.84) \\
467.13 / 1569.05\end{array}$ & $\begin{array}{c}1368.96(342.73) \\
696.93 / 2731.73\end{array}$ & $\begin{array}{l}-0.355(0.29) \\
-1.04 / 0.26\end{array}$ \\
\hline
\end{tabular}

Note. It was found that the overall asymmetry of PT surface was significantly different between the AP mus. group and both the Non-AP mus. group $(P<0.001)$ and the Nonmus. group $(P<0.01)$. No significant difference was found between the Non-AP mus. group and the Nonmus. group in terms of PT asymmetry scores. In terms of surface area, the left PT scores did not differ across any of the groups. However, the right PT surface area was significantly smaller in the AP mus. group compared to both the Non-AP mus. group (P $<0.002)$ and the Nonmus. group $(P<0.006)$. No significant difference was found between the Non-AP mus. group and the Nonmus. group.

as a predictor $\left(\Delta \chi^{2}(1)=9.02, \mathrm{P}<0.003\right.$; final model $=$ $\chi^{2}(2)=22.25$; right PT Wald = 13.37, P $<0.0003$; left PT Wald $=6.79, \mathrm{P}<0.009 ; \%$ class $=81.63 \%$ ).

Using linear regression, it was found that age of commencement was not significantly related to either left $(\mathrm{t}=0.86, \mathrm{P}>0.05)$ or right $\mathrm{PT}(\mathrm{t}=-0.14, \mathrm{P}>$ $0.05)$ surface area $\left(F(2,46)=0.42, P>0.05, R^{2}=0.02\right)$ or overall PT asymmetry $(\mathrm{t}=0.80, \mathrm{P}>0.05 ; \mathrm{F}(2,46)=$ $\left.1.49, P>0.05, R^{2}=0.06\right)$. A final analysis was performed between the current AP group and that previously reported by Schlaug et al. (1995b). It was found that there was no significant difference between the JPT $(\mathrm{t}(36)=0.72, \mathrm{P}>0.05)$.

\section{DISCUSSION}

This study extends and replicates the findings of our previous study demonstrating an increased leftward PT asymmetry in AP musicians (Schlaug et al., 1995b) compared to a matched group of nonmusicians. In addition, our replication is also in agreement with a study by Zatorre and colleagues (1998) in which they compared a small group of AP musicians with a large control group unselected for musical skill. Although the PT asymmetry values were slightly smaller in this study compared to our previous study (Schlaug et al., 1995b), there was no significant difference comparing both cohorts of AP musicians with each other. The fact that these values correspond despite differences in sample size and in the morphometric methods makes a convincing argument for the robustness of the increased leftward PT asymmetry in AP musicians. We have further extended the results of our previous study by testing a group of musicians that had a similar early age of musical training commencement but did not develop absolute pitch.

Results of this study lend support to two critical notions regarding the development of AP. The first relates to early exposure to music. In previous studies it was found that AP manifests itself in a critical period of development, and early exposure to music is paramount to its manifestation (Baharloo et al., 1998; Krumhansl, 1991; Miyazaki, 1988, 1989; Sergeant, 1969). Sergeant (1969) and others found in large samples that almost all musicians with AP started musical training before the age of 7 and found it unlikely that an individual will develop AP if he/she commences musical training after the age of 11 (Sergeant, 1969). By matching the groups on early musical exposure, we have found that with the exception of one person in our AP group, all others had a commencement of musical training on or before the age of 7. However, this exposure in itself was not a determinant of an exaggerated leftward PT asymmetry. It was found that the group of early beginning musicians without AP did have a regular PT asymmetry as can be seen in a nonselected right-handed control group. This finding suggests that in addition to the early commencement of musical training, the increased leftward PT asymmetry is a determinant for the manifestation of AP. However, the effects of music exposure and music training on absolute PT size and degree of asymmetry need to be addressed in a larger sample with more variance in the age of commencement of musical training than in this study. There has been a renewed interest recently in determining genetic correlates of absolute pitch (Baharloo et al., 1998; Gregersen et al., 1999). Although there is no proof yet whether there is one or more genes coding for AP, it is also possible that there is actually a genetic correlate for increased or exaggerated PT asymmetry instead of a genetic correlate for AP. A recent paper (Drayna et al., 2001) found evidence for heritability of pitch recognition in a test in which monozygotic and dizygotic twin pairs were asked to judge whether simple popular melodies contain notes with an incorrect pitch (distorted tunes test). Unfortu- 
nately, musical background was not determined in these twin pairs. Nevertheless, there are many studies that have shown that AP possessors do not differ from non-AP possessors in their pitch judgment ability. The ability to assign any pitch to a pitch class is what sets AP possessors apart from non-AP possessors. Drayna et al. (2001) speculated that AP has a strong environmental component and that it is not clear whether possible genetic factors in AP have any relation to those that underlie pitch recognition ability as tested by the distorted tunes test.

The second critical factor is the nature of the PT asymmetry. The current study extends previous research by suggesting that the exaggerated PT asymmetry observed in AP musicians is predominately driven by a smaller right PT rather than a larger left PT. Galaburda et al., (1987) speculated that the right PT determines the degree of PT asymmetry and that increased asymmetry between homologous areas of the two hemispheres is associated with decreasing number of neurons and decreasing amount of cortex on the smaller side rather than increasing amount on the larger side (Galaburda, 1994). The data reported here appear to indicate that instead of a greater left PT surface or redistribution of resources, the majority of the differences in PT surface can be attributed to right PT scaling. The nature of this is suggested as a pruning of the right PT early in development, though further research will need to be performed to determine if this is in fact the case and to determine whether there is an association between the presumed right PT pruning and the development of AP.

Finally, it is useful to comment on the differences between the morphometric techniques employed and the definition of the regions measured in this and other studies. In contrast to Zatorre's study (Zatorre et al., 1998) we chose to measure PT surface. The main reason for this choice of methods was to remain consistent with our own previous studies which have employed surface measurements, in particular our previous study from 1995 allowing for direct comparisons of PT asymmetry. Determining the PT volume has been problematic with our data and in our experience, since the temporal cortex has a thickness of around $3 \mathrm{~mm}$ and may be represented by maximally 3-4 voxels using the resolution of our MR sequence. The outer voxels may underlie partial volume effects either from cerebral spinal fluid or from white matter. However, cortex volume measurements may be an alternative with the implementation of very high resolution MR data. An improved method may include an average of several acquisitions to achieve a good gray-white differentiation.

A related issue is the anatomical criteria defining the extents of the PT. We have employed criteria that are similar to the original descriptions by Geschwind and Levitsky (1968), which later were adapted by Stein- metz et al . (1991) for use with MR morphometry. These anatomical criteria ensure that only temporal cortex will be included in the PT definition. Westbury et al. (1999) and Steinmetz et al. (1990) performed extensive studies on different ways to quantify the intra-Sylvian surface. The conclusion of both studies was that the typical leftward asymmetry in right-handers and the typical trend toward symmetry in left-handers disappears (a) if one does not include both opposing cortices of the posterior descending ramus of the Sylvian fissure (Steinmetz and Galaburda, 1991) or (b) if one measures the entire temporoparietal surface including the division that has been termed the planum parietale (J äncke and Steinmetz, 1993; L oftus et al ., 1993; Steinmetz et al., 1990, 1991). By using criteria put forward by Steinmetz et al. 1990, 1991), associations between handedness as well as language lateralization have been reported in terms of PT asymmetry (Foundas et al., 1994). Although we used a slightly different method to calculate the true surface in this study compared to our previous studies, we did replicate the main finding of our previous report (Schlaug et al., 1995b). We found no significant difference in the exaggerated $\partial \mathrm{PT}$ in absolute-pitch musicians between the previous and the current report, compared directly.

In summary, it was found that there was a greater leftward asymmetry in the PT of AP musicians compared to non-AP musicians and nonmusicians. The asymmetry appeared to be the result of a smaller right hemisphere PT rather than an increase in the left PT or a distribution of the two. These data suggest that early developmental pruning in the right PT may create an anatomical dominance of the left PT. This in turn might create a functional dominance of the left PT over the right $\mathrm{PT}$, which might be necessary for the acquisition and/or manifestation of absolute pitch. This anatomical difference is likely due to factors other than early musical training or music exposure and it might indicate that young children with an increased leftward PT asymmetry might develop AP if they have an early music exposure.

\section{ACKNOWLEDGMENTS}

The study was supported in part by a grant from the International Foundation for Music Research. Dr. Schlaug is supported by a Doris Duke Clinical Scientist Development Award. Dr. Keenan is now at the Department of Psychology, Montclair State University, Upper Montclair, New J ersey 07043.

\section{REFERENCES}

Annett, M. 1992. Parallels between asymmetries of planum temporale and of hand skill. Neuropsychologia 30: 951-962.

Baharloo, S., J ohnston, P. A., Service, S. K., Gitschier, J ., and Freimer, N. B. 1998. Absolute pitch: An approach for identification of genetic and nongenetic components. Am. J . Hum. Genet. 62: 224231. 
Barta, P. E., Pearlson, G. D., Brill, L. B., 2nd, Royall, R., McGilchrist, I. K., Pulver, A. E., Powers, R. E., Casanova, M. F., Tien, A. Y., Frangou, S., and Petty, R. G. 1997. Planum temporale asymmetry reversal in schizophrenia: Replication and relationship to gray matter abnormalities. Am. J . Psychiatry 154: 661- 667.

Bartels, R. H., Beatty, J . S., and Barsky, B. A. 1989. An Introduction to Splines for Use in Computer Graphics and Geometric Modeling. Morgan Kaufmann, San Mateo, CA.

Besson, M., Faita, F., and Requin, J . 1994. Brain waves associated with musical incongruities differ for musicians and non-musicians. Neurosci. Lett. 168: 101-105.

Bever, T. G., and Chiarello, R. J. 1974. Cerebral dominance in musicians and nonmusicians. Science 185: 537-539.

Drayna D., Manichaikul, A., de Lange, M., Snieder, H., and Spector, T. 2001. Genetic correlates of musical pitch recognition in humans. Science 291: 1969-1972.

Foundas, A. L., Faulhaber, J . R., Kulynych, J . J ., Browning, C. A., and Weinberger, D. R. 1999. Hemispheric and sex-linked differences in Sylvian fissure morphology: A quantitative approach using volumetric magnetic resonance imaging. Neuropsychiatry Neuropsychol. Behav. Neurol. 12: 1-10.

Foundas, A. L., Leonard, C. M., Gilmore, R., Fennell, E., and Heilman, K. M. 1994. Planum temporale asymmetry and language dominance. Neuropsychologia 32: 1225-1231.

Galaburda, A., Corsiglia, J ., Rosen, G., and Sherman, G. 1987. Planum temporale asymmetry, reapprasisal since Geschwind and Levitsky. Neuropsychol ogia 25: 853- 868.

Galaburda, A. M. 1994. Developmental dyslexia and animal studies: At the interface between cognition and neurology. Cognition 50: 133-149.

Geschwind, N., and Levitsky, W. 1968. Human brain: Left-right asymmetries in temporal speech region. Science 161: 186-187.

Gregersen, P. K., Kowalksy, E., Kohn, N., and Marvin, E. W. 1999. Absolute pitch: Prevalence, ethnic variation, and estimation of the genetic component. Am. J . Hum. Genet. 65: 911-913.

Ide, A., Rodriguez, E., Zaidel, E., and Aboitiz, F. 1996. Bifurcation patterns in the human Sylvian fissure: Hemispheric and sex differences. Cereb. Cortex 6: 717-725.

J äncke, L., Schlaug, G., Huang, Y., and Steinmetz, H. 1994. Asymmetry of the planum parietale. NeuroReport 5: 1161-1163.

J äncke, L., Schlaug, G., and Steinmetz, H. 1997. Hand skill asymmetry in professional musicians. Brain Cognit. 34: 424- 432.

J äncke, L., and Steinmetz, H. 1993. Auditory lateralization and planum temporale asymmetry. NeuroReport 5: 169-172.

Kleinschmidt, A., Falkai, P., Huang, Y., Schneider, T., Furst, G., and Steinmetz, H. 1994. In vivo morphometry of planum temporale asymmetry in first-episode schizophrenia. Schizophrenia Res. 12: 9-18.

Krumhansl, C. 1991. Music psychology: Tonal structures in perception and memory. Annu. Rev. Psychol. 42: 277-303.

Kulynych, J . J ., Vladar, K., J ones, D. W., and Weinberger, D. R. 1994. Gender differences in the normal lateralization of the supratemporal cortex: MRI surface-rendering morphometry of Heschl's gyrus and the planum temporale. Cereb. Cortex 4: 107-118.

Leonard, C. M., Voeller, K. K., Lombardino, L. J ., Morris, M. K., Hynd, G. W., Alexander, A. W., Andersen, H. G., Garofalakis, M., Honeyman, J. C., Mao, J ., et al. 1993. Anomalous cerebral structure in dyslexia revealed with magnetic resonance imaging. Arch. Neurol. 50: 461- 469.

Loftus, W. C., Tramo, M. J ., Thomas, C. E., Green, R. L., Nordgren, R. A., and Gazzaniga, M. S. 1993. Three-dimensional quantitative analysis of hemispheric asymmetry in the human superior temporal region. Cereb. Cortex 3: 348-355.
Mazziotta, J . C., Phelps, M. E., Carson, R. E., and Kuhl, D. E. 1982. Tomographic mapping of human cerebral metabolism: Auditory stimulation. Neurology 32: 921-937.

Meyers, D., Skinner, S., and Sloan, K. 1992. Surfaces from contours. ACM Transact. Graphics 11: 228-258.

Miyazaki, K. 1988. Musical pitch identification by absolute pitch possessors. Percept. Psychophys. 44: 501-512.

Miyazaki, K. 1989. Absolute pitch identification: Effects of timbre and pitch region. Music Percept. 7: 1-14.

Schlaug, G., J äncke, L., Huang, Y., Staiger, J . F., and Steinmetz, H. 1995a. Increased corpus callosum size in musicians. Neuropsychologia 33: 1047-1055.

Schlaug, G., J äncke, L., Huang, Y., and Steinmetz, H. 1995b. In vivo evidence of structural brain asymmetry in musicians. Science 267: 699-701.

Sergeant, D. 1969. Experimental investigation of absolute pitch. J . Res. Music Educ. 17: 135-143.

Shapleske, J ., Rossell, S. L., Woodruff, P. W., and David, A. S. 1999. The planum temporale: A systematic, quantitative review of its structural, functional and clinical significance. Brain Res. Brain Res. Rev. 29: 26- 49.

Steinmetz, H., and Galaburda, A. M. 1991. Planum temporale asymmetry: In-vivo morphometry affords a new perspective for neurobehavioral research. Reading Writing 3: 331-343.

Steinmetz, H., Rademacher, J ., Huang, Y. X., Hefter, H., Zilles, K., Thron, A., and Freund, H. J. 1989. Cerebral asymmetry: MR planimetry of the human planum temporale. J . Comput. Assisted Tomogr. 13: 996-1005.

Steinmetz, H., Rademacher, J ., J äncke, L., Huang, Y. X., Thron, A., and Zilles, K. 1990. Total surface of temporoparietal intrasylvian cortex: Diverging left-right asymmetries. Brain Lang. 39: 357372.

Steinmetz, H., Staiger, J . F., Schlaug, G., Huang, Y., and J äncke, L. 1995. Corpus callosum and brain volume in women and men. NeuroReport 6: 1002-1004.

Steinmetz, H., Volkmann, J., J äncke, L., and Freund, H. J . 1991. Anatomical left-right asymmetry of language-related temporal cortex is different in left- and right-handers. Ann. Neurol. 29: 315-319.

Takeuchi, A. H., and Hulse, S. H. 1993. Absolute pitch. Psychol. Bull. 113: 345-361.

Tzourio, N., Crivello, F., Mellet, E., Nkanga-Ngila, B., and Mazoyer, B. 1998. Functional anatomy of dominance for speech comprehension in left handers vs right handers. Neurol mage 8: 1-16.

Ward, W., and Burns, E. 1982. Absolute pitch. In The Psychology of Music (D. Deutsch, Ed.), pp. 431-451. Academic Press, New York.

Westbury, C. F., Zatorre, R. J ., and Evans, A. C. 1999. Quantifying variability in the planum temporale: A probability map. Cereb. Cortex 9: 392-405.

Zatorre, R. J ., and Beckett, C. 1989. Multiple coding strategies in the retention of musical tones by possessors of absolute pitch. Memory Cognit. 17: 582-589.

Zatorre, R. J ., Evans, A. C., and Meyer, E. 1994. Neural mechanisms underlying melodic perception and memory for pitch. J . Neurosci. 14: 1908-1919.

Zatorre, R. J ., Evans, A. C., Meyer, E., and Gjedde, A. 1992. Lateralization of phonetic and pitch discrimination in speech processing. Science 256: 846-849.

Zatorre, R. J ., Perry, D. W., Beckett, C. A., Westbury, C. F., and Evans, A. C. 1998. Functional anatomy of musical processing in listeners with absolute pitch and relative pitch. Proc. Natl. Acad. Sci. USA 95: 3172-3177. 